1. Si l'on doit facturer cet été déjà selon le nouveau tarif dans le domaine de la CNA/AMF/AI, nous devrons, ou plus précisément notre livreur de logiciel devra fabriquer les programmes informatiques nécessaires. Est-ce envisageable?

2. Que signifie exactement "facturation par voie électronique»? Pourra-t-on établir ses factures sur son propre ordinateur ou devra-t-on se connecter à un bureau extérieur?

R. S. $\dot{a} B$.

ad 1: Les conditions techniques sont arrêtées dans leurs principes depuis la mi-1999 et elles seront pleinement opérationnelles le 30 avril 2000. Le problème n'est pas d'ordre technique. Les entreprises de logiciels n'investiront que lorsqu'elles pourront tabler sur la sortie des tarifs TarMed. Ce sera moins du ressort de la FMH que des instances politiques à qui l'on souhaite le meilleur pour s'attaquer enfin aux vrais problèmes et s'atteler à la tâche. Depuis le 2 février 2000, les expédients dont elles ont usé pour s'en soustraire, allant de l'exercice de leur propre incompétence aux assauts répétés contre la FMH appartiennent définitivement au passé.

ad 2: La facture est adressée à l'assureur sur support électronique.
En consultant l'interprétation médicale de la position 00.0030 (prestations de base), mon attention s'est portée sur le passage suivant: "sont comprises ... la remise de médicaments (situation d'urgence et/ou dispensation initiale)...."

Cette formulation me semble remettre en question la propharmacie régulière (ce qui ne répond pas, je l'espère, à une volonté délibérée!) La distribution de médicaments ne saurait se limiter aux situations d'urgence et à une "dispensation initiale».

Je demande que l'on supprime le passage entre parenthèses.

A. S. $\dot{a} M$.

Les médicaments sont également délivrés dans les cantons qui ne pratiquent pas la propharmacie, notamment dans les unités de soins d'urgence, précisément. Cette prestation, pour ce qui concerne la partie médicale, doit être indemnisée par les prestations de base. La propharmacie, quelle qu'en soit la forme (p. ex. la distribution de médicaments indépendante de la marge) n'est aucunement remise en cause par cela. D'ailleurs, l'indemnisation tarifaire prévue dans ce cadre-là fixe une rémunération du médecin pour ses activités dans le cadre de la distribution des médicaments selon le principe des prestations de base.

\title{
Cours de formation
}

\section{à la pratique de l'expertise médicale dans le domaine de l'assurance accidents}

\author{
Montreux, 25 et 26 mai 2000
}

Face à la demande croissante du nombre d'expertise médicales et de la complexité des situations rencontrées pour l'évaluation du dommage corporel et de ses conséquences suite à un accident, la FMH s'est engagée à soutenir l'organisation d'un cours de formation pour les médecins chargés d'établir des expertises médicales dans le domaine de l'assuranceaccidents. Ce cours, également placé sous le patronage de l'Union des sociétés chirurgicales suisses et de la Société suisse de traumatologie et de médecine des assurances, est réalisé en collaboration avec les services médicaux de la SUVA et de l'Association suisse d'assurances (ASA).
D'une durée de 2 jours, ce cours a pour but de permettre d'approfondir les divers aspects médicaux et juridiques de l'expertise médicale, grâce aux concours de divers spécialistes médecins, juristes et juges expérimentés dans le domaine des lésions traumatiques et de la LAA. A cet effet, l'enseignement sera dispensé sous forme d'exposés, de séminaires, d'ateliers interactifs ainsi que d'une table ronde finale. De plus, un support de cours sera remis à chaque participant.

Ce cours qui s'adresse aux médecins détenteurs d'un FMH dans les diverses disciplines médicales sera crédité comme formation continue (16 heures) par les diverses sociétés concernées et chaque participant recevra un certificat de cours. Pour des raisons didactiques et organisationnelles, le nombre de participants sera limité à 50 personnes.

Informations et inscription:

Mme M. Devaud

Secrétariat Centre de Médecine des Assurances

Policlinique médicale universitaire

19, rue César-Roux, 1005 Lausanne

tél. 02134523 04, fax 0213452318

e-mail monique.devaud@hospvd.ch ou

roger.darioli@hospvd.ch 